\title{
Developing a rapid and highly efficient cowpea regeneration and transformation system using embryonic axis explants
}

Ping Che ${ }^{1 *}$, Shujun Chang ${ }^{1,}$, Marissa K. Simon ${ }^{1}$, Zhifen Zhang ${ }^{2}$, Ahmed Shaharyara, Jesse

Ourada ${ }^{1,}$ a , Dennis $\mathrm{O}^{\prime} \mathrm{Neill}^{1}$, Mijael Torres-Mendoza ${ }^{3}$, Yinping Guo², Kathleen M. Marasigan², Jean-Philippe Vielle-Calzada ${ }^{3}$, Peggy Ozias-Akins ${ }^{2}$, Marc C. Albertsen ${ }^{1}$ and Todd J. Jones ${ }^{1}$

${ }^{1}$ Corteva Agriscience, Johnston, lowa 50131 USA.

${ }^{2}$ Department of Horticulture and Institute of Plant Breeding, Genetics \& Genomics, University of Georgia Tifton Campus, Tifton, GA 31973

${ }^{3}$ Group of Reproductive Development and Apomixis, UGA Laboratorio Nacional de Genómica para la Biodiversidad, CINVESTAV Irapuato, Guanajuato 36821, México

*Correspondence Author:

Ping Che

8305 NW 62 ${ }^{\text {nd }}$ Ave

Johnston, IA 50131 USA

Phone: +1-515-535-6105

Email: ping.che@corteva.com

a Present address: Benson Hill Biosystems, 1100 Corporate Square Dr. Suite 150 St. Louis, MO 63132. USA 
Running Title: Cowpea Transformation

Keywords: Cowpea transformation, Embryonic axis, Cotyledonary node (cot-node), Shoot

organogenesis, Spectinomycin, Agrobacterium.

\section{Abbreviations:}

Cot-node: Cotyledonary node; EA: Embryonic axis; SAM: Shoot apical misterm; CTP: Chloroplast

transit peptide; Spec: Spectinomycin; NPTII: Neomycin phosphotransferase II; Kan: kanamycin;

SIM: Shoot induction medium; RIM: Root induction medium; SEM: shoot elongation medium;

Vir: Virulence; NPGS: National Plant Germplasm System. 


\section{Summary}

Cowpea is one of the most important legume crops planted worldwide, especially in SubSaharan Africa and Asia. Despite decades of effort, genetic engineering of cowpea is still challenging due to inefficient in vitro shoot regeneration, Agrobacterium-mediated T-DNA delivery and transgenic selection. Here, we report a rapid and highly efficient cowpea transformation system using embryonic axis explants isolated from imbibed mature seeds. We found that removal of the shoot apical meristem by cutting through the middle of the epicotyl stimulated direct multiple shoot organogenesis from the cotyledonary node tissue.

Furthermore, the application of a ternary transformation vector system using an optimized pVIR accessory plasmid provided high levels of Agrobacterium-mediated gene delivery. The utilization of spectinomycin as the selection agent enabled more efficient transgenic selection and plant recovery. Transgenic cowpea shoots developed exclusively from the cotyledonary nodes at high frequencies of 4.5 to $37 \%$ across a wide range of cowpea genotypes. We believe that the transformation principles established in this study could also be applied to other legumes to increase transformation efficiencies.

\section{Introduction}

Domesticated in Africa and widely cultivated in the tropical and subtropical zones of the world, cowpea (Vigna unguiculata (L.) Walp.), also known as black-eyed pea, is one of the most valuable grain legumes for high-quality dietary protein, carbohydrates, lipids, minerals and vitamins for people in developing countries of Africa and Asia (Abdu Sani et al., 2015; Phillips et 
al., 2003; Singh, 2014). It is estimated that over 200 million people consume cowpea daily in Africa (Phillips et al., 2003; Singh, 2014). Despite its high tolerance to heat, dry conditions and soil acidity, cowpea is highly susceptible to insect pest and pathogen infestations resulting in lower productivity (Abdu Sani et al., 2015; Boukar et al., 2016; Obembe, 2008; Singh, 2014; Solleti et al., 2008a). Due to limited genetic variability of cowpea and strong crossincompatibility between wild Vigna species and cultivated cowpea, little progress has been made in genetic improvement through conventional breeding to achieve desirable agronomic traits (Abdu Sani et al., 2015; Fang et al., 2007; Gomathinayagam et al., 1998; Latunde-Dada, 1990; Wamalwa et al., 2016). Hence, plant biotechnology provides an alternative approach to overcome those production constraints for improving the agronomic performance and developing better cowpea cultivars with higher grain quality and yield (Carlos Popelka et al., 2004; Zaidi et al., 2005). The development of insect-resistant cowpea, unsuccessful through conventional breeding, was successfully achieved by introducing Bt genes through genetic transformation and is a good example of plant biotechnology application in an orphan crop (Bakshi et al., 2011; Bett et al., 2017; Zaidi et al., 2005). Recently, significant progress has been made establishing genomic and gene expression data resources for two cowpea varieties, IT86D-1010 (Spriggs et al., 2018) and IT97K-499-35 (Lonardi et al., 2019; Munoz-Amatriain et al., 2017; Yao et al., 2016). However, the absence of an efficient genetic transformation system (Popelka et al., 2006; Somers et al., 2003) has impeded the full utilization of these resources for cowpea functional genomic studies to elucidate the mechanisms of heat and drought stress tolerance and to improve the agronomic traits, such as insect and pathogen resistances and increased productivity. 
Legumes, especially cowpea, are known to be recalcitrant for genetic manipulation

(Manman et al., 2013; Popelka et al., 2006; Solleti et al., 2008b; Somers et al., 2003). This is due to the absence of an amenable in vitro shoot regeneration system, the inadequate Agrobacterium-mediated T-DNA delivery to the targeted tissue and the inefficient transgenic selection methods for viable transgenic plant recovery. Therefore, published frequencies of Agrobacterium-mediated transformation are lower than 3.9\% (Bett et al., 2019; Chaudhury et al., 2007; Manman et al., 2013; Mellor et al., 2012) and the process often requires more than 5 to 8 months (Chaudhury et al., 2007; Popelka et al., 2006). To overcome those obstacles and improve cowpea transformation efficiency, we evaluated shoot regeneration using embryonic axis (EA) explants isolated from imbibed mature seeds and identified that only cotyledonary node (cot-node) cells of the EA explants undergo rapid cell division and dedifferentiation to acquire organogenetic competence for shoot regeneration. Based on that observation, a rapid and highly efficient in vitro adventitious shoot regeneration system using EA explants was developed for direct de novo shoot organogenesis.

Next, we demonstrated that a ternary vector system developed previously (Anand et al., 2018) provided enhanced gene delivery not only for corn and sorghum transformation as we previously demonstrated (Anand et al., 2018; Che et al., 2018), but also for cowpea transformation using an auxotrophic Agrobacterium strain LBA4404 Thy- (thymidine mutant), a strain that reduces bacterial overgrowth during tissue culture compared to wild-type LBA4404. This ternary vector system contains the T-DNA binary vector and the optimized pVIR accessory (pPHP71539) plasmid with additional vir (Virulence) genes (Anand et al., 2018). The pVIR accessory plasmid and T-DNA binary vector have many desirable features, including smaller 
vector sizes, enhanced vector stability and amended vir genes for enhanced T-DNA gene delivery and ultimately higher transformation efficiency for all crops tested (Anand et al., 2018; Che et al., 2018).

To identify an optimal selection system for the EA-based de novo organogenesis, we also tested and compared the selection efficiency of chloroplast transit peptide (CTP)NPTII/kanamycin (kan), CTP-NPTII/G418 and CTP-spcN (GenBank Accession No. AAD50455)/spectinomycin (spec) (Anada et al., 2017) selectable marker systems based on selection stringency and transgenic plant recovery. We found that the utilization of CTP-spcN combined with spec as the selectable marker facilitated the recovery of transgenic cowpea and provided more efficient and stringent transgenic selection and identification through the transformation process.

Finally, in order to test the robustness and flexibility of the protocol, an alternate spec selection system, CTP-aadA/spec (Martinell et al., 2017), and a hypervirulent Agrobacterium strain AGL1 (Lazo et al., 1991), which was previously used in cowpea transformation (Popelka et al., 2006), were utilized to test the transformability of nine cowpea genotypes for transgenic shoot regeneration and demonstrated the broad application potential of the transformation system.

Overall, we developed a rapid, robust and highly efficient Agrobacterium-mediated EAbased cowpea regeneration, transformation and selection system for generating transgenic cowpea exclusively from the cot-nodes of EA explants at a high frequency, between 4.5 to $37 \%$, across a wide range of cowpea genotypes. 


\section{Results and discussion}

De novo shoot organogenesis using $E A$ as explants

A rapid, efficient and reproducible regeneration system is a prerequisite for establishment of an efficient cowpea genetic transformation system. Although several studies of in vitro regeneration of cowpea based on organogenesis have been reported (Aasim et al., 2010; Abdu Sani et al., 2015; Mamadou et al., 2008; Manman et al., 2013; Odutayo et al., 2005; Raveendar et al., 2009; Sani et al., 2018; Tie et al., 2013; Yusuf et al., 2008), an efficient cowpea regeneration system that enables highly efficient transformation is still lacking (Manman et al., 2013). Soybean transformation based on the preexisting meristems of EA explants has been well established and provides a reliable and highly efficient mean for introducing transgenes (Aragão et al., 2000; Liu et al., 2004; Turlapati et al., 2008). To test the regeneration efficiency of EA explants in cowpea, EA explants were isolated from imbibed mature seeds of cowpea variety IT86D-1010 by excising the cotyledons at the nodal points (Figure 1a). Those EA explants with the plumule excised (Figure 1b) were then cultured directly onto shoot induction medium (SIM) (Table S5) without selection in a vertical upright position with roots embedded in the media to induce shoot development. In most of the cases (>95\%), a single primary shoot was developed per EA when the shoot apical misterm (SAM) of the EA explants was kept intact (non-decapitated EA explants) during regeneration (Figure 1c). However, multiple shoot development was observed occasionally for a small number of explants (<5\%) (Figure $1 \mathrm{~d}$ ). Compared to the morphology of EA explants with single primary shoot development (Figure 1c), the EA explants with multiple shoots, regenerated exclusively around cot-nodes (Figure 1d), 
were much shorter and lacked both epicotyls and SAMs. The lack of epicotyls and SAMs could be due to the accidental damage of those tissues during EA isolation and plumule excision. This finding indicated that the de novo organogenesis of shoots around the cot-nodes could be inhibited by apical dominance. To confirm this hypothesis that multiple shoot development was more efficient from the cot-node tissue, SAMs were purposely removed by cutting through the middle of each epicotyl (decapitated EA explants) (Figure 1a and e) and the isolated EA was cultured for regeneration on SIM. Indeed, removal of the SAM purposely by cutting through the middle of each epicotyl (decapitated EA explants) (Fig. 1a and e) induced $78 \%$ of explants to initiate multiple shoot regeneration in IT86D-1010 (Figure 1f, g and Table 1).

To test if the regeneration principle described above was applicable to other cowpea germplasm accessions and even common bean, we further tested tissue culture and in vitro regeneration procedures for eight additional cowpea accessions from the U.S. National Plant Germplasm System (NPGS), two non-conventional cowpea germplasm lines (TPC-001 and MRS001) and two common bean (Phaseolus vulgaris L) varieties, black bean (CBB-001) and pinto bean (CBP-001), collected from tropical and subtropical regions of Mexico (Figure S3). Consistent with observations in IT86D-1010, shoot regeneration also developed exclusively from cot-node regions for all ten cowpea germplasm lines and two bean germplasm lines tested (Figure S4). In most cases, the number of EA explants showing multiple shoot regeneration exceeded those showing a single regenerated shoot, suggesting that multiple individuals can be recovered from a single EA explant (Table 1 and Figure S4). The overall regeneration efficiency ranged from $55 \%$ to $81 \%$ for eight additional cowpea accessions from the NPGS, 36 to $38 \%$ for Mexican cowpeas and $30 \%$ for common beans (Table 1). These results demonstrated that tissue 
culture and regeneration procedures can be applied to a wide collection of cowpea germplasm and extended to other legumes such as common bean.

\section{Regeneration optimization under Agrobacterium-mediated transformation}

Generally, the EA-based dicot transformation procedure consists of the following main steps: explant preparation, Agrobacterium infection, co-cultivation, shoot regeneration with selection and root induction (Figure S2. Also see Experimental Procedures for detail). As described above, although EA explants per se have been described for soybean transformation, the cowpea EAbased regeneration system has a key difference. While the soybean EA transformation system relies on preexisting apical meristematic tissue for regeneration and transformation (Aragão et al., 2000; Liu et al., 2004; Turlapati et al., 2008), the cowpea system shows that removal of the SAM stimulated multiple shoot organogenesis from the cot-node. This key difference raises the question of how well the decapitated-EA explants will be able to survive and regenerate throughout the Agrobacterium-mediated transformation procedure.

To evaluate how the decapitation of EA explants affects the survival and regeneration capability, we conducted sonication, Agrobacterium infection and co-cultivation treatments, (Figure S2. Also see Experimental Procedures for detail) either with or without Agrobacterium, followed by regeneration on SIM. This allowed us to measure the survival and regeneration of the decapitated and non-decapitated EA explants and assess possible damage caused by the early steps of transformation, such as sonication-associated wounding, potential medium component toxicity, sensitivity to Agrobacterium and the length of the treatment steps before regeneration. As shown in Figure 2a, the decapitated EA explants were extremely sensitive to 
the treatments and none of the EA explants survived on SIM without selection after mimicking all the treatment steps without Agrobacterium infection. On the contrary, all the nondecapitated EA explants survived and formed elongated epicotyls with a single primary shoot. The further decapitation of those primary shoots by cutting through the middle of the elongated epicotyls after 4-days of regeneration stimulated multiple shoot organogenesis around the cot-nodes (Figure $2 \mathrm{~b}$ ). Collectively, those observations suggest that although the SAM negatively regulates multiple shoot organogenesis from cot-node tissue because of the apical dominance effect, the SAM is essential for EA explant survival through the transformation treatments before regeneration and the SAM should not be removed until fully recovered after 4 days of regeneration.

It has been reported that Agrobacterium-mediated infection leads to cell damage and tissue necrosis (Norkunas et al., 2018). To determine the survival rate of EA explants after infection and co-cultivation with Agrobacterium, non-decapitated EA explants were subjected to the transformation procedure (Figure S2. Also see Experimental Procedures for transformation procedure) using LBA4404 Thy- carrying the pPHP86170/pPHP71539 vector system as described below. As shown in Figure 2c, about $70 \pm 10 \%$ EA explants (based on the average of 3 replicates and total 75 EA explants) survived and formed elongated epicotyls after 4-day regeneration on SIM with selection (Table S5). Compared to the $100 \%$ survival rate without Agrobacterium inoculation (Figure $2 \mathrm{~b}$ ), the $30 \%$ rate of the explant loss was most likely due to the sensitivity of EA explants to the Agrobacterium. 
Based on those studies, the decapitation of EA explants after 4 or 5 days of regeneration on SIM was routinely performed for all the subsequent transformation optimization experiments throughout this study.

Agrobacterium-mediated gene delivery using ternary vector system

A dramatic increase of T-DNA delivery efficiency was reported in cowpea by constitutive expression of additional vir genes in a resident pSB1 vector in Agrobacterium strain LBA4404 (Solleti et al., 2008b). Recently, we demonstrated that a newly designed ternary vector containing the T-DNA binary vector and the optimized pVIR accessory (pPHP71539) plasmid with additional vir genes enhanced gene delivery and ultimately the transformation efficiency for both corn and sorghum (Anand et al., 2018; Che et al., 2018). This encouraged us to assess the gene delivery efficiency of the ternary vector for cowpea transformation.

To evaluate T-DNA delivery using the ternary vector system in cowpea, we transformed binary vector pPHP86170 (Figure S1a) containing the proDMMV:TagRFP as visual marker and proGM-UBQ:CTP-spcN as the selectable marker into the Agrobacterium strain LBA4404 Thyharboring the pVIR accessory plasmid pPHP71539. Transient gene delivery was assessed by visually evaluating the number of fluorescent foci on the surface of cowpea EA explants after 3 days of shoot induction on SIM containing $25 \mathrm{mg} / \mathrm{l} \mathrm{spec}$ as selection. As shown in Figure 3b, strong gene delivery based on the number of infected cells was visualized across the entire explant for those surviving EA explants with elongated epicotyls (Figure 3a and b), especially around the cot-node tissue (Figure $3 b$ and $c$ ), demonstrating highly efficient gene delivery in cowpea EA explants using the ternary vector system. Although gene delivery was efficient 
across the entire EA explant, only those fluorescent foci within the cot-node tissue showed subsequent development and substantially enhanced fluorescence intensity during regeneration (Figure $3 \mathrm{~b}$ and $\mathrm{c}$ ). This observation supported the hypothesis that only those cells within the cot-node tissue, but not any other tissues of the EA explant, actively undergo rapid cell division and dedifferentiation to acquire organogenetic competence for shoot regeneration.

In vitro regeneration and transgenic selection of cowpea

Several selection systems have been reported for cowpea transformation with different explant types (Manman et al., 2013), such as NPTII/kan (Bett et al., 2019; Chaudhury et al., 2007), NPTII/G418 (Solleti et al., 2008b), PMI/mannose (Bakshi et al., 2012), HPT/hygromycin (Kumar et al., 1996), BAR/glufosinate (Popelka et al., 2006) and ahas/imazapyr (Citadin et al., 2013; Ivo et al., 2008). It was reported that incomplete selection and tissue necrosis were associated with those selection systems and resulted in lower transgenic plant recovery in cowpea (Bakshi et al., 2011; Chaudhury et al., 2007; Solleti et al., 2008b). To identify more efficient selection agents for the de novo organogenesis described above, we tested and compared the selection efficiency of CTP-NPTII/kan, CTP-NPTII/G418 and CTP-spcN/spec after Agrobacterium-mediated transformation using Agrobacterium strain LBA4404 Thy-. We found that the utilization of CTPspcN/spec system provided more efficient and stringent transgenic selection than either CTPNPTII/kan or CTP-NPTII/G418 and without non-transgenic escapes and chimeric tissue formation. As described above, strong gene delivery was observed around the cot-node target tissue on the third day of regeneration as shown in Figure $3 b$ and c. Those fluorescent foci grew 
quickly and single or multiple shoot buds emerged exclusively around the cot-nodes within 2 weeks following removal of the SAM on the fourth day of regeneration (Figure $3 \mathrm{~d}$ and e). Transgenic shoots were fully developed from the buds within another 3 weeks in which all the shoots displayed strong fluorescence evenly across the entire regenerated shoot (Figure $3 f$ and g) compared to the regenerated shoots from wild-type cowpea IT86D-1010 EA explants that showed no fluorescence at all (Figure S5 a and b). The elongated shoots were excised from the EA explants and transferred to root induction medium (RIM) (Table S6) for root development. Because of the stringent selection during shoot organogenesis, selection was not required for rooting. Approximately 95\% (Table 2 ) of the elongated shoots fully rooted in the RIM (Table S6) within 2-3 weeks and all the regenerated shoots and roots displayed strong fluorescence (Figure $3 \mathrm{~h}$ and i). The total time from inoculation of the EA explants to transplantation of a fully developed transgenic plantlet in the greenhouse took approximately $2-3$ months. The frequency of shoot formation was about $21 \%$ for IT86D-1010, of which about $23 \%$ of the events were single-copy quality events (Table 2) (see Experimental Procedures for "quality events" definition and event quality determination).

Conversely, cowpea IT86D-1010 EA explants showed a high degree of resistance to kan as a selection agent and no selection pressure could be built up by culturing non-transformed EA explants on SIM containing kan at concentrations as high as $600 \mathrm{mg} / \mathrm{l}$. Although, an optimal concentration of G418 at $20 \mathrm{mg} / \mathrm{l}$ for selection of transformed shoots was established by culturing non-transformed EA explants on SIM containing different concentrations of G418 (10$40 \mathrm{mg} / \mathrm{l}$ ), the G418 selection was not as stringent as spec and only chimeric events (Figure S6a and b) were observed using the binary vector pPHP94518 (Figure S1b) containing proGM- 
EF1A2:Ds-RED as a visual marker and proGM-UBQ:CTP-NPTII as the selectable marker. Chimera formation was indicated by uneven and partial fluorescence of the regenerated shoot in Figure S6a and $b$.

Transgene inheritance in the progeny

To evaluate the inheritance of T-DNA integration events, we selected three independent singlecopy quality T0 transgenic events in the IT86D-1010 background (see Experimental Procedures for "quality events" definition and event quality determination) transformed with construct pPHP92782 (Figure S1c) containing the proGM-EF1A2:Ds-RED as a visual marker gene and proGM-UBQ:CTP-spcN as the selectable marker. Those T0 plants were self-pollinated in the greenhouse and the resultant T1 seeds displayed either red color (similar seed color with DsRED expression was reported in soybean (Nishizawa et al., 2006)) or no visual pigmentation difference from the wild-type as shown in Figure S7a and b, demonstrating the transmission and segregation of the transgene in the progeny. To further determine the segregation ratio, one hundred T1 seeds from each of the three independent events were randomly chosen regardless of seed color and advanced to the T1 generation. The zygosity (homozygous, hemizygous and null) of individual T1 plants was characterized by determining the copy number of the integrated T-DNA based on the assays described in the Experimental Procedures. As shown in Table 3, the segregation pattern of these transgenic events showed typical 1:2:1 and 3:1 Mendelian ratio based on Chi-square test $\left(\chi^{2}\right)$ for all the copy number assays performed (Figure S1c and Table S9). Those results demonstrate that all the transgenic events analyzed 
possessed stably integrated T-DNA and the T-DNA was faithfully inherited to the next generation.

Transformability evaluation of different cowpea genotypes

Genotype dependence is a major limitation of regeneration and Agrobacterium-mediated transformation for both monocots and dicots (Abdu Sani et al., 2015; Che et al., 2018; Jia et al., 2015; Manman et al., 2013). To evaluate the robustness of the protocol and broaden the application of this cowpea transformation technology for different cowpea genotypes, we performed a quick transformability assay to evaluate the formation of fluorescent transgenic shoots after 2 weeks of culture on SIM with selection. This quick transformability assay was conducted using a hypervirulent Agrobacterium strain AGL1 carrying RC2717 plasmid with CTP$\operatorname{aad} A$ as the selectable marker (Figure S1d). As shown in Table 4, transgenic shoot regeneration frequency (defined as transformability) of IT86D-1010 determined by the quick transformability assay was in a range of 11 to $26 \%$ (average $19 \% \pm 7.5 \%$ ) (Table 4 ). This was comparable to the $21 \% \pm 2 \%$ transformation efficiency described earlier using LBA4404 Thy- carrying the pVIR accessory plasmid for transformation and CTP-spcN/spec for selection (Table 2), indicating the reliability of this quick assay for predicating transformation efficiency of different genotypes. The application of this quick transformability assay to eight more cowpea genotypes showed the transformability in a range of 4.5 to $37 \%$. Similar to the observation of transgenic shoot development for IT86D-1010, all the eight cowpea genotypes also formed transgenic shoots exclusively and rapidly at the cot-node region and, in most of the cases, no more than two transgenic shoots per explants were developed (Figure S8). These results demonstrate that the 
transformation protocol developed for IT86D-1010 described herein is transferable to other genotypes even with an alternative Agrobacterium-mediated transformation system.

In general, better shoot organogenesis response tends to produce higher transgenic shoot regeneration frequency, but this is not always the case. As shown in Table 4 and 1, although all five genotypes, IT86D-1010 and PI 527675, PI 580227, PI 582835 and TVu 79, showed very good and comparable shoot organogenesis response in the range of 78 to $81 \%$ efficiency, only IT86D-1010, PI 527675 and PI 582835 demonstrated significant high transformability (19\% $\pm 7.5 \%, 31 \%$ and $37 \%$, respectively), but not PI 580227 and TVu 79 (5.6\% and 4.5\%, respectively). In contrast, all three genotypes, TVu 3562, TVu 9693 and PI 583259, had relatively low shoot organogenesis response (68\%, $61 \%$ and $56 \%$, respectively) (Table 1 ), but their transformability was relatively high (18\%, $22 \%$ and $26 \%$, respectively) (Table 4). Therefore, the transformability of those germplasm lines is determined not only by the shoot organogenesis capability, but also by the combination of the susceptibility to Agrobacteriummediated T-DNA delivery and sensitivity to the Agrobacterium infection and sonication related damages.

Taken together, we have developed a rapid, robust, flexible and highly efficient cowpea transformation system using EA as explants. The principles established in this study have the potential to increase the transformation efficiencies of other legume species and, potentially, other dicot crops. With recent progress establishing cowpea genetic and genomic resources (Lonardi et al., 2019; Munoz-Amatriain et al., 2017; Spriggs et al., 2018; Yao et al., 2016), we 
believe that the broad application of this cowpea transformation system will have immediate and far-reaching impact on cowpea research that will improve cowpea productivity.

\section{Experimental Procedures}

Agrobacterium strain and vectors

Two Agrobacterium strains, the auxotrophic strain LBA4404 Thy- and AGL1, were used in this study. Agrobacterium auxotrophic strain LBA4404 Thy- was used with the ternary vector transformation system for cowpea IT86D-1010 transformation. The ternary vector system contains the T-DNA binary vector and the optimized pVIR accessory (pPHP71539) plasmid as previously described by Che et al. (2018) and Anand et al (2018). The T-DNA binary plasmid pPHP86170 (Figure S1a) contains the PUC ORI, the NPTIII bacterial selectable marker, the TagRFP reporter gene and $\operatorname{spcN}$ (Anada et al., 2017) as plant selectable marker gene. The binary plasmid pPHP94518 (Figure S1b) contains the PVS1 ORI, the spec bacterial selectable marker, the Ds-RED reporter gene and NPTII as plant selectable marker gene. The binary plasmid pPHP92782 contains the PVS1 ORI, the NPTIII bacterial selectable marker, the Ds-RED reporter gene, spcN (Anada et al., 2017) as plant selectable marker gene (Figure S1c). The ternary design was assembled by first mobilizing the accessory plasmid pPHP71539 in the Agrobacterium auxotrophic strain LBA4404 Thy- and selected on media supplemented with gentamycin $(25 \mathrm{mg} / \mathrm{l})$. Subsequently the binary constructs were electroporated into Agrobacterium strain LBA4404 Thy- containing the accessory plasmid and recombinant colonies were selected on media supplemented with gentamycin plus either kan for pPHP86170 and pPHP92782 or spec 
for pPHP94518. All constructs were then subjected to next generation sequencing and sequence confirmation before conducting transformation experiments.

AGL1 carrying RC2717, a modified pCAMBIA vector (Figure S1d), was used for testing transformation on different cowpea germplasm other than IT86D-1010. The T-DNA region of the binary vector RC2717 contained a soybean-codon-optimized aadA1 gene (Martinell et al., 2017) as a selectable marker and a reporter cassette in which a TdTomato reporter cassette flanked by two loxP sites was placed in a reversed orientation between a soybean-codonoptimized ZsGreen gene and the Arabidopsis Ubiquitin 10 promoter (Figure S1d). The TdTomato gene provided a visible fluorescence marker to identify transgenic shoots after transformation. With the use of the freeze-thaw method (Chen et al., 1994), the binary vector was introduced into AGL1 and the recombinant colonies were selected on medium containing $100 \mathrm{mg} / \mathrm{l} \mathrm{kan}$.

Materials reported in this paper may contain components subject to third party ownership (e.g., TagRFP and Ds-RED). Transgenic and genome edited materials may be subject to governmental regulations. Availability of materials described in this paper to academic investigators for non-commercial research purposes under an applicable material transfer agreement will be subject to proof of permission from any third-party owners of all or parts of the material and to governmental regulation considerations. Obtaining the applicable permission from such third-party owners will be the responsibility of the requestor. Transgenic materials reported in this paper may only be made available if in full accordance with all applicable governmental regulations. 
Plant materials and growth conditions

Cowpea varieties IT86D-1010, PI 527675, PI 580227, PI 582835, PI583259, TVu 8670, TVu 3562,

TVu 9693, and TVu 79 originally obtained from the U.S. NPGS (https://www.ars-grin.gov/npgs/)

were used for this study. Mexican cowpea accessions TPC1-001 and MRS-001 were collected

from local farming communities of Tabasco and Morelos States, respectively; common bean

(Phaseolus vulgaris $\mathrm{L}$ ) varieties black bean (CBB-001) and pinto bean (CBP-001) were obtained

from local producers in Guanajuato State. Those varieties were maintained in the greenhouse

to collect mature seeds for EA explant isolation.

Cowpea transformation procedure

The main steps of cowpea transformation mediated by Agrobacterium were illustrated in Figure

S2. The detailed cowpea transformation protocol including Agrobacterium preparation, cowpea

EA isolation, transformation procedure and medium preparation were described in the

Supporting information.

Microscopy and imaging

Images were taken using a dissecting Leica M165 FC stereo-epifluorescence microscope, with

RFP and Ds-RED filters for detection of fluorescence, using the PLANAPO 1.0x objective, 0.63x

zoom, and Leica Application Suite V4.7 acquisition software. The autofluorescence of the wild-

type regenerated cowpea was evaluated using the same system. For testing transformation on

the eight additional cowpea germplasm lines from NPGS, transgenic shoots expressing

TdTomato were monitored with a Stemi SVII dissection stereoscope equipped with a HBO

illuminator (Carl Zeiss, Thornwood, NY) and a Ds-RED filter (excitation: 545/25 nm, emission:

605/70 nm, Chroma Technology, Bellows Falls, VT). Images were taken by using an AxioCam 
camera (Carl Zeiss, Oberkochen, Germany) and the AxioVision LE64 software, and composed by using Photoshop CC (Adobe, San Jose, CA).

\section{Evaluation of transgenic plants}

The integrated copy number of the T-DNA of the binary vector in the transgenic plants was determined by a series of Quantitative PCR ( $q P C R$ ) analyses based on the method previously described by Wu et al. (2014). In this study, an endogenous control qPCR assay (LBS) (Table S9) was developed using a house-keeping gene annotated as 3-isopropy/malate dehydrogenase (Vigun05g298700) in the leucine biosynthetic pathway from cowpea (Misra et al., 2017). Four qPCR assays (PINII_TERM, sPCN_SO, CTP and UBQ14_TERM) for pPHP86170 (Figure S1a and Table S9) and two qPCR assays (Ds-RED, spcN_SO) for pPHP92782 (Figure S1c and Table S9) were developed to determine T-DNA copy number by normalizing with endogenous control LBS assay.

Outside the border integration sites, PCR backbone-specific assays were developed to check for any border read-through (Wu et al., 2014). The presence and absence of Agrobacterium vector backbone integration of the binary vector was detected based on screening for sequences from three regions outside of the T-DNA integration sites for each vector, such as SPC, LEFTBORDER and NPTIII for plasmid pPHP92782 and HYG, VIRG and HYGROMYCIN for plasmid pPHP86170 (Figure S1a, c and Table S9).

Stable T-DNA integration was confirmed by copy number determination using genomic DNA extracted from the putative TO transgenic events. The TO transgenic plants carrying a single-copy of the intact T-DNA integrations without vector backbone for all assays described were defined as quality events (Anand et al., 2018; Che et al., 2018; Zhi et al., 2015). The 
percentage of quality events was divided by the total number of events analyzed to calculate the quality event frequency. Only quality events were advanced to the greenhouse for the next generation. The zygosity of the T1 plants was established by determining the copy number of the T-DNA for all the event quality assays (Figure S1a, c and Table S9). Chi-square analysis $\left(\chi^{2}\right)$ was performed to determine whether the difference between the observed and expected ratio was statistically significant.

\section{Acknowledgements}

We thank the super binary vector construction team from Corteva Agriscience for their support with vector construction and environmental control group for cowpea planting in the greenhouse. We thank Dr. Hyeon-Je Cho from Corteva Agriscience for developing the soybean transformation procedure which provided instrumental knowledge for this study. We also thank Drs. Ray Collier and Michael Petersen from the Wisconsin Crop Innovation Center for constructing the RC2717 vector. The study was funded by a sub-award from the CSIRO to Corteva, UGA and LANGEBIO under the Capturing Heterosis for Smallholder Farmers grant from the Bill and Melinda Gates Foundation (BMGF). Corteva Agriscience provided funding and inkind donations for this project as well. 


\section{Author contributions}

P.C., S.C., M.S., M.A. and T.J. conceptualized the methods. P.C., S.C., M.S., Z.Z., J.V., P.O., M.A.

and T.J. designed research, analyzed the data and wrote the paper. A.S. conducted cowpea

transformation and collected the data for IT86D-1010. Z.Z. Y.G. and K.M. conducted

transformation and genotyping for other cowpea genotypes collected from NPGS. J.O. and D.O.

developed event quality assays. M.T. conducted regeneration experiment for Mexican cowpeas

and beans.

Conflict of Interest: The authors have no conflict of interest to declare.

\section{References}

Aasim, M., Khawar, K.M. and Özcan, S. (2010) Efficient in vitro propagation from preconditioned embryonic axes of Turkish cowpea (Vigna unguiculata L.) cultivar Akkiz. Archives of Biological Sciences 62, 1047-1052.

Abdu Sani, L., Usman, I.S., Ishiaku Faguji, M. and Muhammad Bugaje, S. (2015) Towards Efficient In vitro Regeneration of Cowpea (Vigna unguiculata L. Walp): A Review. British Biotechnology Journal 7, 174-182.

Anada, A., Bass, S.H., Bertain, S.M., Cho, H.-J., Kinney, A.J., Klein, T.M., Lassner, M., Mcbride, K.E., Moy, Y., Rosen, B.A.M. and Wei, J.-Z. (2017) Ochrobactrum-mediated transformation of plants. (WO/2017/040343 ed).

Anand, A., Bass, S.H., Wu, E., Wang, N., McBride, K.E., Annaluru, N., Miller, M., Hua, M. and Jones, T.J. (2018) An improved ternary vector system for Agrobacterium-mediated rapid maize transformation. Plant molecular biology 97, 187-200.

Aragão, F.J.L., Sarokin, L., Vianna, G.R. and Rech, E.L. (2000) Selection of transgenic meristematic cells utilizing a herbicidal molecule results in the recovery of fertile transgenic soybean [Glycine max (L.) Merril] plants at a high frequency. Theoretical and Applied Genetics 101, 1-6.

Bakshi, S., Sadhukhan, A., Mishra, S. and Sahoo, L. (2011) Improved Agrobacterium-mediated transformation of cowpea via sonication and vacuum infiltration. Plant cell reports $30,2281-$ 2292. 
Bakshi, S., Saha, B., Roy, N.K., Mishra, S., Panda, S.K. and Sahoo, L. (2012) Successful recovery of transgenic cowpea (Vigna unguiculata) using the 6-phosphomannose isomerase gene as the selectable marker. Plant cell reports 31, 1093-1103.

Bett, B., Gollasch, S., Moore, A., Harding, R. and Higgins, T.J.V. (2019) An Improved Transformation System for Cowpea (Vigna unguiculata L. Walp) via Sonication and a Kanamycin-Geneticin Selection Regime. Frontiers in plant science 10, 219.

Bett, B., Gollasch, S., Moore, A., James, W., Armstrong, J., Walsh, T., Harding, R. and Higgins, T.J.V. (2017) Transgenic cowpeas (Vigna unguiculata L. Walp) expressing Bacillus thuringiensis Vip3Ba protein are protected against the Maruca pod borer (Maruca vitrata). Plant Cell, Tissue and Organ Culture (PCTOC) 131, 335-345.

Boukar, O., Fatokun, C.A., Huynh, B.L., Roberts, P.A. and Close, T.J. (2016) Genomic Tools in Cowpea Breeding Programs: Status and Perspectives. Frontiers in plant science 7, 757.

Carlos Popelka, J., Terryn, N. and Higgins, T.J.V. (2004) Gene technology for grain legumes: can it contribute to the food challenge in developing countries? Plant Science 167, 195-206.

Chaudhury, D., Madanpotra, S., Jaiwal, R., Saini, R., Kumar, P.A. and Jaiwal, P.K. (2007) Agrobacterium tumefaciens-mediated high frequency genetic transformation of an Indian cowpea (Vigna unguiculata L. Walp.) cultivar and transmission of transgenes into progeny. Plant Science 172, 692-700.

Che, P., Anand, A., Wu, E., Sander, J.D., Simon, M.K., Zhu, W., Sigmund, A.L., Zastrow-Hayes, G., Miller, M., Liu, D., Lawit, S.J., Zhao, Z.Y., Albertsen, M.C. and Jones, T.J. (2018) Developing a flexible, high-efficiency Agrobacterium-mediated sorghum transformation system with broad application. Plant biotechnology journal 16, 1388-1395.

Chen, H., Nelson, R.S. and Sherwood, J.L. (1994) Enhanced recovery of transformants of Agrobacterium tumefaciens after freeze-thaw transformation and drug selection. BioTechniques 16, 664-668, 670.

Citadin, C.T., Cruz, A.R. and Aragao, F.J. (2013) Development of transgenic imazapyr-tolerant cowpea (Vigna unguiculata). Plant cell reports 32, 537-543.

Fang, J., Chao, C.-C.T., Roberts, P.A. and Ehlers, J.D. (2007) Genetic diversity of cowpea [Vigna unguiculata (L.) Walp.] in four West African and USA breeding programs as determined by AFLP analysis. Genetic Resources and Crop Evolution 54, 1197-1209.

Gomathinayagam, P., Ganesh ram, S., Rathnaswamy, R. and Ramaswamy, N.M. (1998) Interspecific hybridization between Vigna unguiculata (L.) Walp. and V. vexillata (L.) A. Rich. through in vitro embryo culture. Euphytica 102, 203-209.

Ivo, N.L., Nascimento, C.P., Vieira, L.S., Campos, F.A. and Aragao, F.J. (2008) Biolistic-mediated genetic transformation of cowpea (Vigna unguiculata) and stable Mendelian inheritance of transgenes. Plant cell reports 27, 1475-1483.

Jia, Y., Yao, X., Zhao, M., Zhao, Q., Du, Y., Yu, C. and Xie, F. (2015) Comparison of Soybean Transformation Efficiency and Plant Factors Affecting Transformation during the Agrobacterium Infection Process. Int J Mol Sci 16, 18522-18543.

Kumar, M., Mariamma, M., Veluthambi, K. and Gnanam, A. (1996) Genetic transformation of cotyledon explants of cowpea (Vigna unguiculata L. Walp) using Agrobacterium tumefaciens. Plant Cell Rep. 15, 980-985.

Latunde-Dada, A.O. (1990) Genetic Manipulation of the Cowpea (Vigna unguiculata [L.] Walp.) for Enhanced Resistance to Fungal Pathogens and Insect Pests. In: Advances in Agronomy (Brady, N.C. ed) pp. 133-154. Academic Press.

Lazo, G.R., Stein, P.A. and Ludwig, R.A. (1991) A DNA transformation-competent Arabidopsis genomic library in Agrobacterium. Bio/technology 9, 963-967. 
Liu, H.K., Yang, C. and Wei, Z.M. (2004) Efficient Agrobacterium tumefaciens-mediated transformation of soybeans using an embryonic tip regeneration system. Planta 219, 1042-1049.

Lonardi, S., Munoz-Amatriain, M., Liang, Q., Shu, S., Wanamaker, S.I., Lo, S., Tanskanen, J., Schulman, A.H., Zhu, T., Luo, M.C., Alhakami, H., Ounit, R., Hasan, A.M., Verdier, J., Roberts, P.A., Santos, J.R.P., Ndeve, A., Dolezel, J., Vrana, J., Hokin, S.A., Farmer, A.D., Cannon, S.B. and Close, T.J. (2019) The genome of cowpea (Vigna unguiculata [L.] Walp.). The Plant journal : for cell and molecular biology 98, 767-782.

Mamadou, S., Diallo, Ndiaye, A., Sagna, M. and Kène Gassama-Dia, Y. (2008) Plants regeneration from African cowpea variety (Vigna unguiculata L. Walp.). African Journal of Biotechnology 7, 28282833.

Manman, T., Qian, L., Huaqiang, T., Yongpeng, Z., Jia, L.F. and Huanxiu, L. (2013) A review of regeneration and genetic transformation in cowpea (Vigna unguiculata L. Walp). African Journal of Agricultural Research 8, 1115-1122.

Martinell, B.J., Petersen, M.W., Somers, D.A., Wan, Y., Williams, E. and Ye, X. (2017) Methods for plant transformation using spectinomycin selection. In: US8466345B2. United States: MONSANTO TECHNOLOGY LLC (St. Louis, MO, US).

Mellor, K.E., Hoffman, A.M. and Timko, M.P. (2012) Use of ex vitro composite plants to study the interaction of cowpea (Vigna unguiculata L.) with the root parasitic angiosperm Striga gesnerioides. Plant methods 8, 22-22.

Misra, V.A., Wang, Y. and Timko, M.P. (2017) A compendium of transcription factor and Transcriptionally active protein coding gene families in cowpea (Vigna unguiculata L.). BMC genomics 18, 898.

Munoz-Amatriain, M., Mirebrahim, H., Xu, P., Wanamaker, S.I., Luo, M., Alhakami, H., Alpert, M., Atokple, I., Batieno, B.J., Boukar, O., Bozdag, S., Cisse, N., Drabo, I., Ehlers, J.D., Farmer, A., Fatokun, C., Gu, Y.Q., Guo, Y.N., Huynh, B.L., Jackson, S.A., Kusi, F., Lawley, C.T., Lucas, M.R., Ma, Y., Timko, M.P., Wu, J., You, F., Barkley, N.A., Roberts, P.A., Lonardi, S. and Close, T.J. (2017) Genome resources for climate-resilient cowpea, an essential crop for food security. The Plant journal : for cell and molecular biology 89, 1042-1054.

Nishizawa, K., Kita, Y., Kitayama, M. and Ishimoto, M. (2006) A red fluorescent protein, DsRed2, as a visual reporter for transient expression and stable transformation in soybean. Plant cell reports 25, 1355-1361.

Norkunas, K., Harding, R., Dale, J. and Dugdale, B. (2018) Improving agroinfiltration-based transient gene expression in Nicotiana benthamiana. Plant Methods 14, 71.

Obembe, O.O. (2008) Exciting times for cowpea genetic transformation research. Life Sci J 5, 50-52.

Odutayo, O.I., Akinrimisi, F.B., Ogunbosoye, I. and Oso, R.T. (2005) Multiple shoot induction from embryo derived callus cultures of cowpea (Vigna unguiculata I.) Walp. African Journal of Biotechnology 4, 1214-1216.

Phillips, R.D., McWatters, K.H., Chinnan, M.S., Hung, Y.-C., Beuchat, L.R., Sefa-Dedeh, S., Sakyi-Dawson, E., Ngoddy, P., Nnanyelugo, D., Enwere, J., Komey, N.S., Liu, K., Mensa-Wilmot, Y., Nnanna, I.A., Okeke, C., Prinyawiwatkul, W. and Saalia, F.K. (2003) Utilization of cowpeas for human food. Field Crops Research 82, 193-213.

Popelka, J.C., Gollasch, S., Moore, A., Molvig, L. and Higgins, T.J. (2006) Genetic transformation of cowpea (Vigna unguiculata L.) and stable transmission of the transgenes to progeny. Plant cell reports 25, 304-312.

Raveendar, S., Premkumar, A., Sasikumar, S., Ignacimuthu, S. and Agastian, P. (2009) Development of a rapid, highly efficient system of organogenesis in cowpea Vigna unguiculata (L.) Walp. South African Journal of Botany 75, 17-21. 
Sani, L.A., Usman, I.S., Ishiyaku, M. and Bugaje, S.M. (2018) Optimization of protocol for multiple shoots regeneration from apical meristem of embryonic axes in cowpea (Vigna unguiculata L. Walp). Bayero Journal of Pure and Applied Sciences 10, 428-433.

Singh, B.B. (2014) Cowpea: The Food Legume of the 21st Century. Madison, WI: Crop Science Society of America, Inc. DOI: 10.2135/2014.cowpea.

Solleti, S.K., Bakshi, S., Purkayastha, J., Panda, S.K. and Sahoo, L. (2008a) Transgenic cowpea (Vigna unguiculata) seeds expressing a bean alpha-amylase inhibitor 1 confer resistance to storage pests, bruchid beetles. Plant cell reports 27, 1841-1850.

Solleti, S.K., Bakshi, S. and Sahoo, L. (2008b) Additional virulence genes in conjunction with efficient selection scheme, and compatible culture regime enhance recovery of stable transgenic plants in cowpea via Agrobacterium tumefaciens-mediated transformation. Journal of biotechnology 135, 97-104.

Somers, D.A., Samac, D.A. and Olhoft, P.M. (2003) Recent advances in legume transformation. Plant physiology 131, 892-899.

Spriggs, A., Henderson, S.T., Hand, M.L., Johnson, S.D., Taylor, J.M. and Koltunow, A. (2018) Assembled genomic and tissue-specific transcriptomic data resources for two genetically distinct lines of Cowpea ( Vigna unguiculata (L.) Walp). Gates open research 2, 7.

Tie, M., Luo, Q., Zhu, Y. and Li, H. (2013) Effect of 6-BA on the Plant Regeneration via Organogenesis from Cotyledonary Node of Cowpea (Vigna unguiculata L. Walp). Journal of Agricultural Science 5, 1-5.

Turlapati, S.A., S.K, J., M R, B. and P.B, K. (2008) Cotyledonary Node and Embryo Axes as Explants in Legume Transformation with Special Reference to Peanut. In: Handbook of New Technologies for Genetic Improvement of Legumes pp. 253-271.

Wamalwa, E.N., Muoma, J. and Wekesa, C. (2016) Genetic Diversity of Cowpea (Vigna unguiculata (L.) Walp.) Accession in Kenya Gene Bank Based on Simple Sequence Repeat Markers. International journal of genomics 2016, 8956412.

Wu, E., Lenderts, B., Glassman, K., Berezowska-Kaniewska, M., Christensen, H., Asmus, T., Zhen, S., Chu, U., Cho, M.J. and Zhao, Z.Y. (2014) Optimized Agrobacterium-mediated sorghum transformation protocol and molecular data of transgenic sorghum plants. In vitro cellular \& developmental biology. Plant : journal of the Tissue Culture Association 50, 9-18.

Yao, S., Jiang, C., Huang, Z., Torres-Jerez, I., Chang, J., Zhang, H., Udvardi, M., Liu, R. and Verdier, J. (2016) The Vigna unguiculata Gene Expression Atlas (VuGEA) from de novo assembly and quantification of RNA-seq data provides insights into seed maturation mechanisms. The Plant journal : for cell and molecular biology 88, 318-327.

Yusuf, M., A. Raji, A., Ingelbrecht, I. and D. Katung, M. (2008) Regeneration efficiency of cowpea (Vigna unguiculata (L.) Walp.) via embryonic axes explants. African Journal of Plant Science 2, 105-108.

Zaidi, M.A., Mohammadi, M., Postel, S., Masson, L. and Altosaar, I. (2005) The Bt gene cry2Aa2 driven by a tissue specific ST-LS1 promoter from potato effectively controls Heliothis virescens. Transgenic research 14, 289-298.

Zhi, L., TeRonde, S., Meyer, S., Arling, M.L., Register, J.C., 3rd, Zhao, Z.Y., Jones, T.J. and Anand, A. (2015) Effect of Agrobacterium strain and plasmid copy number on transformation frequency, event quality and usable event quality in an elite maize cultivar. Plant cell reports 34, 745-754. 


\section{Figure Legends}

Figure 1 The regeneration origin of cowpea EA explants via organogenesis. (a) The structure of cowpea IT86D-1010 EA extracted from imbibed mature seed. The red line through the middle of epicotyl represents the decapitation process of EA explant to remove the SAM. (b-d) The single (c) and multiple (d) shoot development from non-decapitated EA explants (b). (e-f) The multiple shoot regeneration (f) from decapitated EA explants (e). (g) Close look of multiple shoot regeneration and the cutting site of the epicotyl indicated by the arrow. The percentages in pictures (c, $d$ and $f$ ) represent the population of single and multiple shoot development determined from 100 non-decapitated and 100 decapitated EA explants after 10 days on SIM.

Figure 2 Explant sensitivity to the infection and co-cultivation treatments. (a-b) The survival and regeneration capability of decapitated (a) and non-decapitated (b) EA explants after 10-day shoot induction without selection following infection and co-cultivation steps without Agrobacterium inoculation. (c) The susceptibility of non-decapitated EA explants to the Agrobacterium inoculation following infection and co-cultivation steps. Image was taken on 4day SIM. Arrow and circle indicates one of the survived explants with elongated epicotyl and one of the dying explant without elongated epicotyl, respectively.

Figure 3 Different stages of transgenic cowpea IT86D-1010 development using CTP-spcN/spec selection system. (a-b) T-DNA delivery determined by transient assay after 3-day shoot induction. (c) Close look the number, size and intensity of fluorescent foci around the cot-node region. (d-e) Transgenic shoot budding after 2-week regeneration on SIM with spec selection. (f-g) Fully developed transgenic shoot after 5-week regeneration with spec selection. (h-i) Root 
development of regenerated transgenic shoot after 3-week root induction on RIM. (a, d, f and h) Bright field images. (b, c, e, g and i) Fluorescence images under RFP filter.

\section{Supporting information}

Additional Supporting Information may be found online in the supporting information tab for this article:

\section{Cowpea transformation protocol}

Figure S1 Schematic representation of the molecular components of constructs used in this study.

Figure S2 Flow diagram of the cowpea EA-based Agrobacterium-mediated transformation process.

Figure S3 Dry mature seeds of selected accessions of cowpea and common bean.

Figure S4 Shoot organogenesis of selected accessions of cowpea and common bean.

Figure S5 Autofluorescence evaluation. (a) The bright field image and (b) fluorescence image under RFP filter of regenerated wild-type cowpea IT86D-1010. The arrow indicates the regenerated roots.

Figure S6 Development of chimeric event using CTP-NPTII/G418 selection system. (a) Bright field image. (b) Fluorescence image under RFP filter. 
Figure S7 Transgene segregation in the progeny. (a) Mature wild-type cowpea IT86D-1010 seeds. (b) Segregated T1 seeds in IT86D-1010 background harvested from T0 plant containing the proGM-EF1A2:Ds-RED as visual marker.

Figure S8 Formation of transgenic shoots expressing TdTomato on the EA explants of nine cowpea germplasm lines after $14-d$ culture on SIM, bar $=1 \mathrm{~mm}$.

Table S1 Master plate medium

Table S2 Working plate medium

Table S3 Infection medium (IM)

Table S4 Bean germination medium (BGM)

Table S5 Shoot induction medium (SIM)

Table S6 Root induction medium (RIM)

Table S7 Shoot elongation medium (SEM)

Table S8 OMS

Table S9 Primers used for event quality assays 
bioRxiv preprint doi: https://doi.org/10.1101/738971; this version posted August 19,2019 . The copyright holder for this preprint (which was not certified by peer review) is the author/funder, who has granted bioRxiv a license to display the preprint in perpetuity. It is made available under aCC-BY-NC-ND 4.0 International license.

Table 1 Shoot organogenesis of selected accessions of cowpea and common bean

\begin{tabular}{lcccc}
\hline $\begin{array}{l}\text { Germplasm } \\
\text { accessions }\end{array}$ & \# of EAs & \# of EAs with single shoot (\%) & \# of EAs with multiple shoots (\%) & Explants forming shoots (\%) \\
\hline $\begin{array}{l}\text { Cowpea } \\
\text { IT86D-1010 }\end{array}$ & 36 & $0(0)$ & $28(78)$ & 78 \\
PI 527675 & 36 & $0(0)$ & $29(81)$ & 81 \\
PI 580227 & 36 & $2(6)$ & $26(72)$ & 78 \\
PI 582835 & 29 & $1(3)$ & $22(76)$ & 79 \\
PI 583259 & 36 & $3(8)$ & $17(47)$ & 56 \\
TVu 8670 & 36 & $2(6)$ & $22(61)$ & 67 \\
TVu 3562 & 37 & $4(11)$ & $21(57)$ & 68 \\
TVu 9693 & 36 & $0(0)$ & $22(61)$ & 61 \\
TVu 79 & 36 & $1(3)$ & $28(78)$ & 81 \\
Mexican cowpea & & & & $12(24)$ \\
TPC-001 & 50 & $6(12)$ & $14(28)$ & 36 \\
MRS-001 & 50 & $5(10)$ & & 38 \\
Common bean & & & $11(22)$ & $8(16)$ \\
CBB-001 & 50 & $4(8)$ & & 30 \\
CBP-001 & 50 & $6(12)$ & & 30 \\
\hline
\end{tabular}

Table 2 Transformation efficiency and event quality for IT86D-1010

\begin{tabular}{lccccc}
\hline \# of EAs & $\begin{array}{c}\text { Transgeni } \\
\text { c shoots }\end{array}$ & $\begin{array}{c}\text { Transgenic shoots } \\
\text { with root developed }\end{array}$ & $\begin{array}{c}\text { Rooting } \\
\text { efficiency (\%) }\end{array}$ & $\begin{array}{c}\text { Transformation } \\
\text { efficiency (\%) }\end{array}$ & Quality events (\%) \\
\hline 30 & 7 & 6 & 86 & 20 & 23 \\
30 & 7 & 7 & 100 & 23 & \\
20 & 4 & 4 & 100 & 20 & \\
Average* & & & $95 \pm 8$ & $21 \pm 2$ & \\
\hline
\end{tabular}

Table 3 Segregation analysis of self-fertilized IT86D-1010 transgenic cowpea plant in the T1

generation

\begin{tabular}{lcccccc}
\hline Event ID & $\begin{array}{c}\text { Total plants } \\
\text { analyzed }\end{array}$ & $\begin{array}{c}\text { \# of Two-copy } \\
\text { plants* }\end{array}$ & $\begin{array}{c}\text { \# of single-copy } \\
\text { plants* }\end{array}$ & $\begin{array}{c}\text { \# of null } \\
\text { plants* }\end{array}$ & $P\left(\chi^{2} 1: 2: 1\right)$ & $P\left(\chi^{2} 3: 1\right)$ \\
\hline 125739938 & 100 & 17 & 54 & 29 & $0.17(3.52)$ & $0.36(0.85)$ \\
125739949 & 98 & 28 & 37 & 33 & $0.04(6.39)$ & $0.05(3.93)$ \\
125739950 & 99 & 17 & 57 & 25 & $0.17(3.57)$ & $0.95(0.0033)$ \\
\hline
\end{tabular}

*Copy numbers were determined by both Ds-RED and $s p c N-S O$ qPCR assays. 
bioRxiv preprint doi: https://doi.org/10.1101/738971; this version posted August 19,2019 . The copyright holder for this preprint (which was not certified by peer review) is the author/funder, who has granted bioRxiv a license to display the preprint in perpetuity. It is made available under aCC-BY-NC-ND 4.0 International license.

\section{Table 4 Transformability evaluation of nine cowpea accessions}

\begin{tabular}{lccc}
\hline Germplasm & \# of EAs & \# of EAs with fluorescent shoots in 2 weeks & Transformability (\%) \\
\hline Exp 1 & & & \\
IT86D-1010 & 123 & 14 & 11 \\
PI 527675 & 166 & 52 & 31 \\
PI 580227 & 178 & 10 & 5.6 \\
TVu 9693 & 160 & 35 & 22 \\
\hline Exp 2 & & & \\
IT86D-1010 & 117 & 22 & 19 \\
PI 583259 & 119 & 31 & 26 \\
TVu 79 & 157 & 7 & 4.5 \\
TVu 8670 & 125 & 11 & 8.8 \\
\hline Exp 3 & & & \\
IT86D-1010 & 102 & 27 & 26 \\
PI 582835 & 90 & 33 & 37 \\
TVu 3562 & 177 & 32 & 18 \\
\hline
\end{tabular}


bioRxiv preprint doi: $\mathrm{https}$ //doi.org/10.1101/738971; this version posted August 19, 2019. The copyright holder for this preprint (which was not certified by peer review) is the author/funder, who has granted bioRxiv a license to display the preprint in perpetuity. It is made available under aCC-BY-NC-ND 4.0 International license.

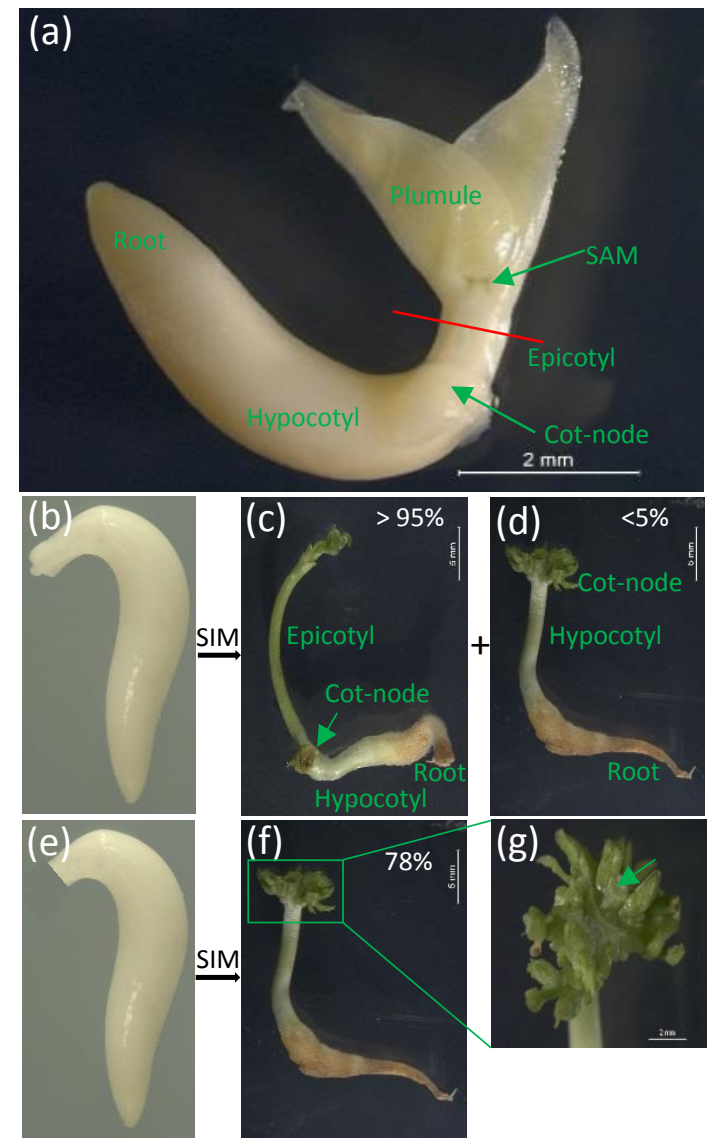

Figure 1 The regeneration origin of cowpea EA explants via organogenesis.

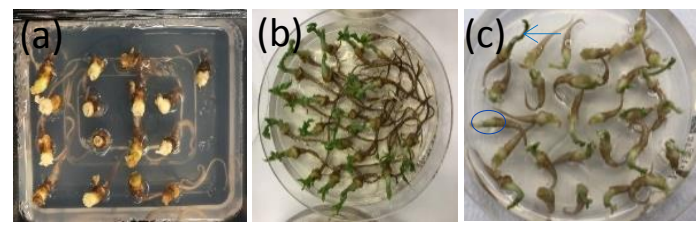

Figure 2 Explant sensitivity to the infection and co-cultivation treatments. 
bioRxiv preprint doi: https://doi.org/10.1101/738971; this version posted August 19,2019 . The copyright holder for this preprint (which was not certified by peer review) is the author/funder, who has granted bioRxiv a license to display the preprint in perpetuity. It is made available under aCC-BY-NC-ND 4.0 International license.

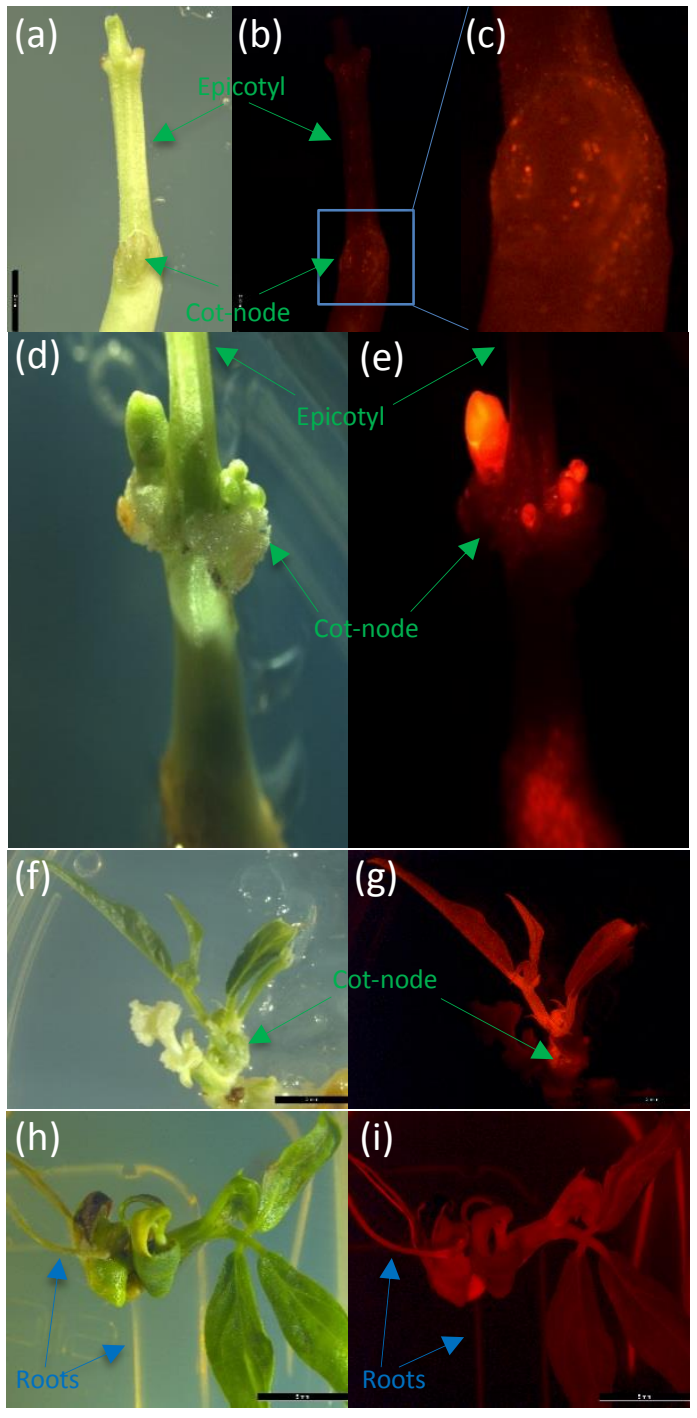

Figure 3 Different stages of transgenic cowpea IT86D-1010 development using CTP-spcN/spec selection system. 